Old Dominion University

ODU Digital Commons

Physics Faculty Publications

Physics

2021

\title{
One-Dimensional Lateral Force Anisotropy at the Atomic Scale in Sliding Single Molecules on a Surface
}

\author{
Yuan Zhang \\ Old Dominion University, y7zhang@odu.edu \\ Daniel J. Trainer \\ Badri Narayanan \\ Yang Li \\ Anh T. Ngo
}

See next page for additional authors

Follow this and additional works at: https://digitalcommons.odu.edu/physics_fac_pubs

Part of the Atomic, Molecular and Optical Physics Commons, and the Tribology Commons

\section{Original Publication Citation}

Zhang, Y., Trainer, D. J., Narayanan, B., ... Curtiss, L. A., Sankaranarayanan, S., \& Hla, S. W. (2021). Onedimensional lateral force anisotropy at the atomic scale in sliding single molecules on a surface. Nano Letters, 21(15), 6391-6397. https://doi.org/10.1021/acs.nanolett.0c04974

This Article is brought to you for free and open access by the Physics at ODU Digital Commons. It has been accepted for inclusion in Physics Faculty Publications by an authorized administrator of ODU Digital Commons. For more information, please contact digitalcommons@odu.edu. 


\section{Authors}

Yuan Zhang, Daniel J. Trainer, Badri Narayanan, Yang Li, Anh T. Ngo, Sushila Khadka, Arnab Neogi,

Brandon Fisher, Larry A. Curtiss, Subramanian K.R.S. Sankaranarayanan, and Saw Wai Hla 


\title{
One-Dimensional Lateral Force Anisotropy at the Atomic Scale in Sliding Single Molecules on a Surface
}

\author{
Yuan Zhang, ${ }^{\bigcirc}$ Daniel J. Trainer, ${ }^{\bigcirc}$ Badri Narayanan, Yang Li, Anh T. Ngo, Sushila Khadka, Arnab Neogi,
} Brandon Fisher, Larry A. Curtiss, Subramanian K. R. S. Sankaranarayanan, and Saw Wai Hla*

Cite This: Nano Lett. 2021, 21, 6391-6397

Read Online

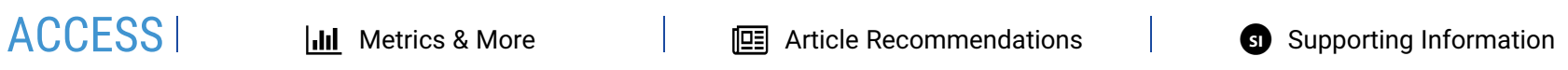

ABSTRACT: Using a $\mathrm{q}+$ atomic force microscopy at low temperature, a sexiphenyl molecule is slid across an atomically flat $\operatorname{Ag}(111)$ surface along the direction parallel to its molecular axis and sideways to the axis. Despite identical contact area and underlying surface geometry, the lateral force required to move the molecule in the direction parallel to its molecular axis is found to be about half of that required to move it sideways. The origin of the lateral force anisotropy observed here is traced to the one-dimensional

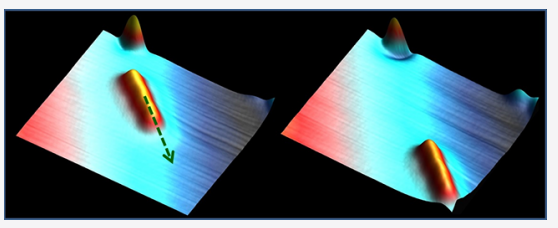
shape of the molecule, which is further confirmed by molecular dynamics simulations.

We also demonstrate that scanning tunneling microscopy can be used to determine the comparative lateral force qualitatively. The observed one-dimensional lateral force anisotropy may have important implications in atomic scale frictional phenomena on materials surfaces.

KEYWORDS: Lateral Force, Friction, Scanning Tunneling Microscopy, Atomic Force Microscopy, Sexiphenyl, Ag(111)

$\mathrm{T}$ he lateral force needed to move a molecule on a surface is important for understanding dynamics as well as related tribological phenomena at the atomic and molecular scales. With the advancement of surface science techniques, such as atomic force microscopy (AFM) $)^{1-9}$ and scanning tunneling microscopy (STM) ${ }^{10,11}$ it has become possible to gain deeper insight into the lateral forces and corresponding tribological phenomena of the atoms, molecules, and nanoscale systems on surfaces. Although a myriad of studies have been conducted to elucidate the friction at the nano- and atomic scale, ${ }^{12-17}$ many tribological phenomena are yet to be understood. ${ }^{18,19}$ At the atomic scale, the potential energy landscape of surfaces plays a critical role in friction. ${ }^{20}$ While barrier height diminishes under a constant load in friction force microscopy, dynamic force microscopy enables the investigation of barrier height contributions to friction. ${ }^{21}$ Since barrier height dictates dynamics of individual atoms and molecules on surfaces, measuring the magnitude of lateral force required to move them on a surface will impact the fundamental understanding of friction at the single molecule level on periodic atomic potential surfaces. Here, we unravel one-dimensional anisotropic behavior in lateral forces to move a molecule on a two-dimensional symmetric surface using a $q+$ atomic force microscope $(\mathrm{q}+\mathrm{AFM})$ tip. Additionally, we show that scanning tunneling microscopy can be used to qualitatively determine the lateral force required to move a molecule on a surface.

Consider an atomically flat area of the single crystal surface of $\operatorname{Ag}(111)$, which has a close-packed surface atomic layer with trigonal symmetry. On this surface, an equal magnitude of lateral force is expected to move an adsorbate along six equivalent directions formed by the surface close-packed atomic rows. ${ }^{11}$ This is the case for lateral movement of individual atoms simply due to the surface symmetry and the zero-dimensional nature of the adsorbed atom (Figure 1a). But for a one-dimensional (1D) adsorbate, moving it along six equivalent close-packed atomic rows involves two directions; one is parallel to its long axis (parallel direction), and the other is $60^{\circ}$ with respect to its long axis (sideways direction). If the adsorbate obeyed the microscopic laws of friction, ${ }^{15}$ the lateral force required to move it would be the same regardless of direction as its contact area with the substrate is always the same (Figure 1a). Here, we demonstrate that this is not necessarily the case at the atomic scale.

We chose para-sexiphenyl (6P) molecules for this study because they have a representative $1 \mathrm{D}$ shape. $6 \mathrm{P}$ is composed of six $\pi$-rings connected as a linear chain (Figure $1 \mathrm{~b}$ ), and it is widely investigated due to potential applications in optoelectronic devices. $^{22-26}$ The experiments were performed using a low-temperature Createc $\mathrm{GmbH}$ system in the $\mathrm{q}+\mathrm{AFM}$ modality for lateral force measurements and the STM modality for lateral manipulation. ${ }^{27}$ For the substrate, a $\operatorname{Ag}(111)$ single crystal surface was cleaned by repeated cycles of $\mathrm{Ar}+$ ion sputtering and annealing to $\sim 650 \mathrm{~K}$. $6 \mathrm{P}$ molecules were deposited onto the atomically clean $\operatorname{Ag}(111)$ substrate via thermal evaporation using a custom built Knudsen cell. The sample was then transferred to the $\mathrm{q}+\mathrm{AFM}$ or STM scanner under ultrahigh

Received: December 17, 2020

Revised: June 7, 2021

Published: July 20, 2021 

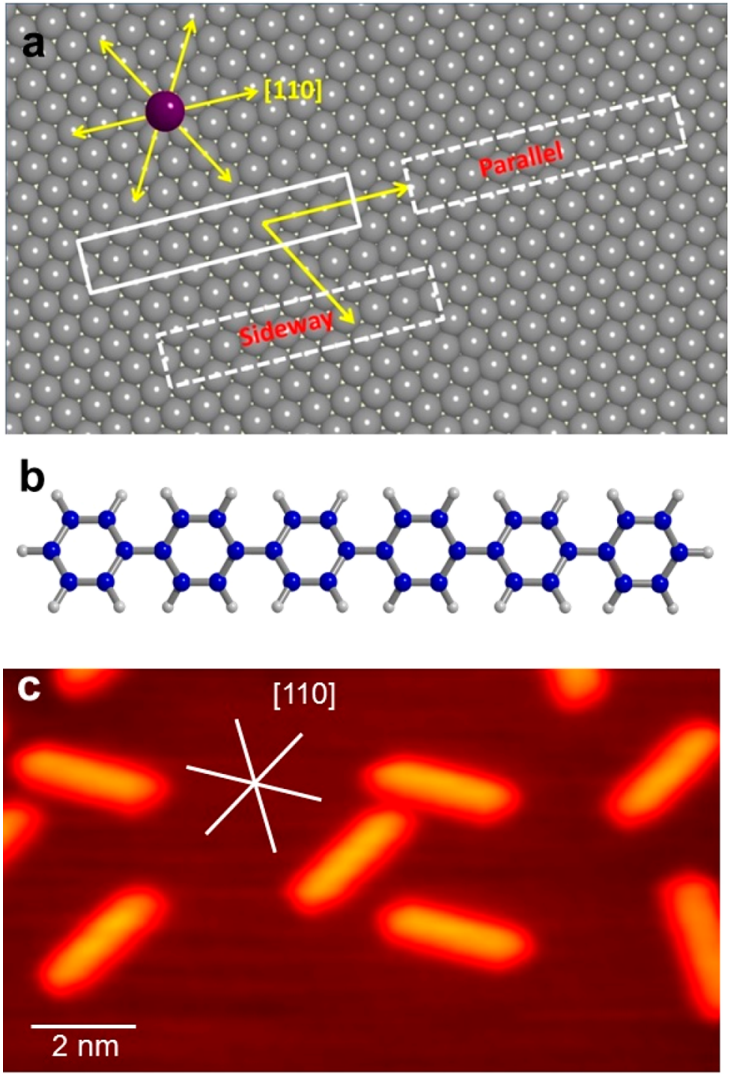
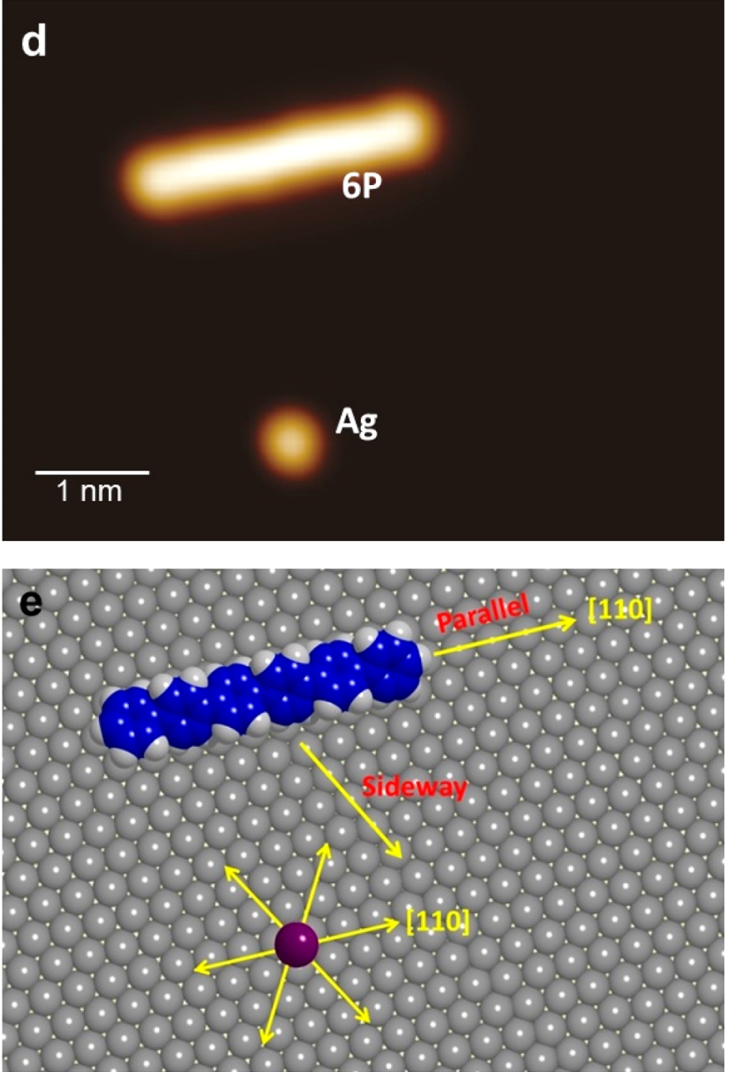

Figure 1. (a) A drawing depicting two different shaped adsorbates on $\mathrm{Ag}(111)$ : an atom as a symmetric ball and a rectangular adsorbate. The dashed rectangles depicts after moving along parallel and sideway directions on $\mathrm{Ag}(111)$. (b) A ball and stick model of 6P. (c) An STM image showing individual 6P molecules adsorbed on a $\mathrm{Ag}(111)$ surface at $5 \mathrm{~K}\left[13.3 \times 7.6 \mathrm{~nm}^{2}, I_{\mathrm{t}}=1.0 \times 10^{-10} \mathrm{~A}, V_{\mathrm{t}}=-2 \mathrm{~V}\right]$. (d) STM image of a sexiphenyl molecule and a silver atom on a $\mathrm{Ag}(111)$ surface $\left[6.3 \times 5.0 \mathrm{~nm}^{2}, \mathrm{I}_{\mathrm{t}}=4.9 \times 10^{-10} \mathrm{~A}, \mathrm{~V}_{\mathrm{t}}=0.48 \mathrm{~V}\right]$. (e) A drawing illustrating a $6 \mathrm{P}$ molecule and a silver atom on a $\mathrm{Ag}(111)$ surface. The yellow arrows indicate lateral movement directions across the surface using scanning probe tip.

vacuum (UHV) condition and cooled down to $\sim 5 \mathrm{~K}$ for the measurements. In STM images, the molecule appears as a 2.7 $\mathrm{nm}$ long rodlike structure on the $\mathrm{Ag}(111)$ surface (Figure 1c), and its long molecular axis is adsorbed parallel to the surface close-packed rows, ${ }^{22}$ that is, the [110] surface directions. Figure $1 \mathrm{~d}$,e shows an STM image and corresponding schematic drawing of a $6 \mathrm{P}$ molecule and a silver adatom on $\operatorname{Ag}(111)$ surface for a comparison.

First, the lateral force measurements were performed with a $\mathrm{q}$ +AFM setup using a procedure demonstrated by Ternes et al. ${ }^{1} \mathrm{q}$ + AFM operates as a noncontact AFM where a tip is attached to a tuning fork with a high " $Q$ " value that vibrates at a certain frequency. To measure the lateral force required to move a $6 \mathrm{P}$ molecule in the parallel direction, the tip with an oscillation amplitude of $3.25 \mathrm{~nm}$ is scanned over a $4 \mathrm{~nm}$ line directly above the molecule along its long molecular axis (Figure 2a) while recording the change in sensor frequency (df) as a function of lateral position $(x)$. Initially, the tip is scanned at a larger height and then the tip height is gradually reduced at subsequent scans. When the tip approaches the molecule, the tip-sample force increases. This leads to change in the sensor frequency and corresponding vertical stiffness of the tuning fork $\left(K_{z}\right)$. This procedure is repeated until the molecule is laterally moved from its initial position. The lateral movement of the molecule is confirmed by acquiring a topographic image (Figure 2b). From each scan of df versus $x$, the changes in vertical stiffness of the tuning fork can be determined. In order to remove the contribution of van der Waals forces, the background is subtracted and the resultant $K_{z}$ versus $x$ scans are plotted as a function of the tip height (Figure 2c). As the tip height is reduced, a dip in $K_{z}$ starts to appear over the molecule due to its interaction with the tip until finally a discontinuity occurs, which is associated with the lateral displacement of the $6 \mathrm{P}$ molecule. The vertical force curves $\left(F_{z}\right)$ are deconvoluted using the SaderJarvis method (Figure 2d, and Supporting Information). ${ }^{28}$ The potential $\left(U_{z}\right)$ is then generated by integrating the vertical force curves for each tip height (Figure 2e). The lateral force component $\left(F_{\mathrm{L}}\right)$ is then extracted by differentiating $U_{z}$ along the lateral distance, $x$ (Figure 2f). The magnitude of the lateral force $\left(F_{\mathrm{Lp}}\right)$ required to move the $6 \mathrm{P}$ molecule along the parallel direction is determined to be $118.8 \pm 15.8 \mathrm{pN}$. Next, this procedure is repeated for the sideways displacement of $6 \mathrm{P}$ as shown in Figure $2 \mathrm{~g}-1$. Here the tip is scanned for $4 \mathrm{~nm}$ along the surface close-packed direction, which is $60^{\circ}$ with respect to the long molecular axis (Figure $2 \mathrm{~g}$ ). The lateral force $\left(F_{\mathrm{Ls}}\right)$ required to move the $6 \mathrm{P}$ molecule in the sideways direction is determined to be $252.4 \pm 21.6 \mathrm{pN}$. To confirm the validity of the $\mathrm{q}+\mathrm{AFM}$ force measurements, we have performed an inflection point test $^{29,30}$ with the results showing that the measurements are conducted in the well-posed regime (see Supporting Information). From the measured force values, the ratio of required lateral force between the parallel and sideways directions $\left(F_{\mathrm{Ls}}\right)$ $\left.F_{\text {Lp }}\right)$ is determined to be $\sim 2.1$. Thus, these measurements indicate that the shape of the molecule plays a vital role in the lateral force required to move the molecule where it takes about 

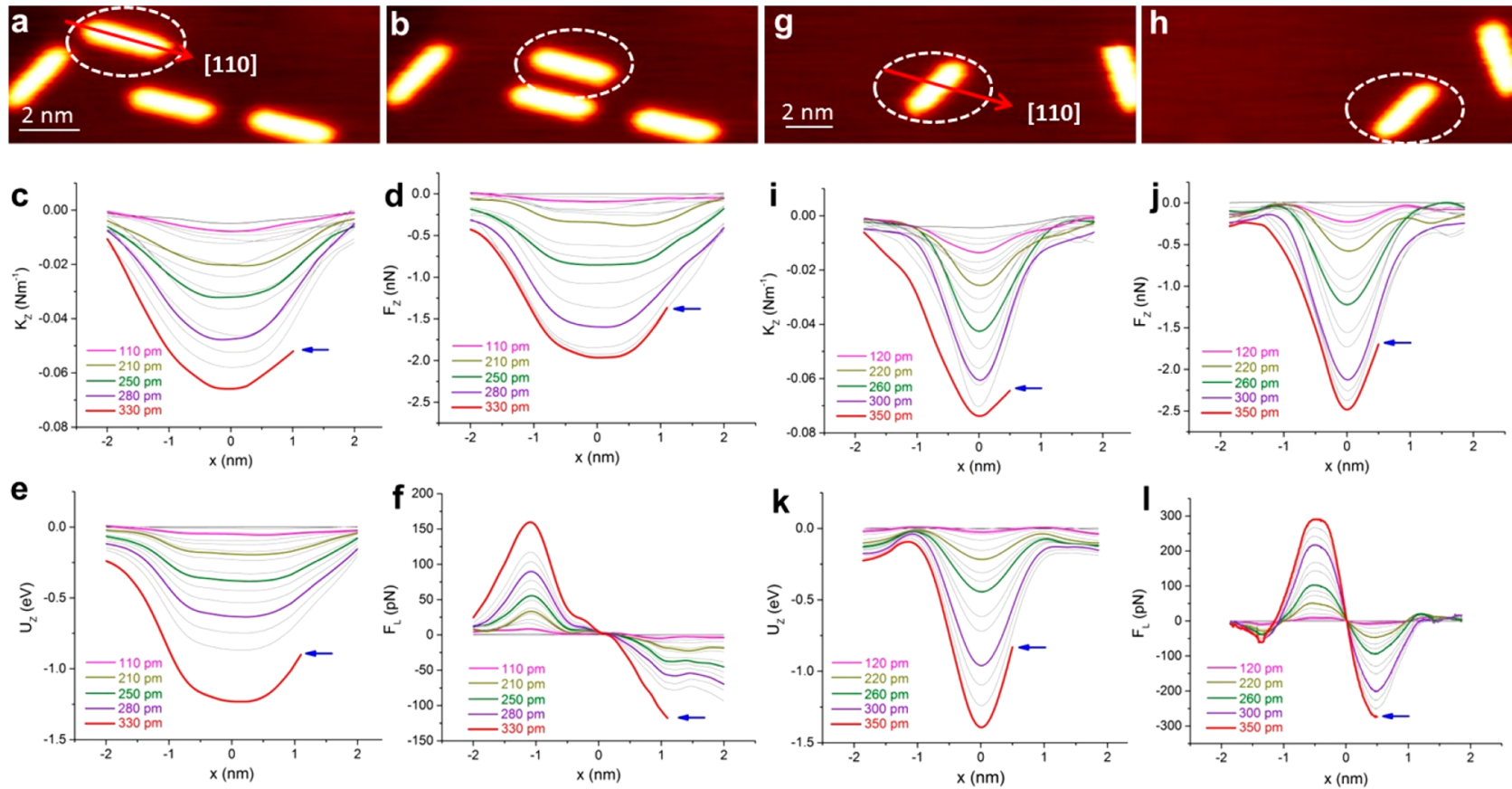

Figure 2. (a) A STM image of 6P molecules on $\mathrm{Ag}(111)$. The molecule is laterally moved to the direction parallel to its long molecular axis (indicated with an arrow) with the q+AFM tip. (b) The image after the manipulation confirms its lateral displacement. (c) Vertical stiffness as a function of lateral distance. Repeated plots are recorded at different tip heights as labeled. (d,e) Vertical force $\left(F_{z}\right)$ and potential $\left(U_{z}\right)$ as a function of lateral distance plots for different tip heights, respectively. (f) Lateral force as a function of lateral distance. (g,h) STM images of before and after manipulation of a 6P molecule along a sideway direction (indicated with an arrow). The dashed ovals in the images mark the manipulated molecule. (i,j,k) The vertical stiffness $\left(K_{z}\right)$, vertical force $\left(F_{z}\right)$, and potential $\left(U_{z}\right)$ functions of lateral distance for the sideway manipulation. (1) Lateral force as a function of lateral distance for sideway manipulation. The color coded labels indicate the vertical tip approach distance from the initial tip height on $\mathrm{Ag}(111)$. Arrows in $(\mathrm{c}-\mathrm{f}, \mathrm{i}-\mathrm{l})$ indicate discontinuity occurs upon the molecule movement.

twice as much force to move the molecule sideways as it does parallel.

We supplement the q+AFM quantification of lateral force with STM lateral manipulation, which offers a straightforward method to observe the displacement of the manipulated molecules. STM lateral manipulation of 6P molecules (Figure 3) are performed by using a tunneling resistance ranging from 0.1 to $2 \mathrm{M} \Omega .^{31,32}$ Here, the STM tip height is reduced from 3 to $4 \AA$ toward the molecule to enhance the tip molecule interaction, and then the tip is scanned over the molecule along the surface close-packed row directions. For the parallel direction, the molecule is moved by pulling the front $\pi$-ring (Figure $3 \mathrm{a}$ ). For the sideways directions, the molecule is moved from the center as well as front $\pi$-ring positions (Figure $3 \mathrm{~d}$ ). We find the same result regardless of the tip positions over the molecule for the sideways manipulations (see Supporting Information). During this process, the molecule moves across the surface together with the tip, and the corresponding tip height signal is recorded. At the final location, the tip is retracted back to the imaging height while the molecule is left on the surface. Successful lateral displacement of the molecule is confirmed by an STM topographic image acquired after the manipulation (Figure $3 \mathrm{~b}, \mathrm{e})$. In both the parallel and sideways directions, the $6 \mathrm{P}$ molecule moves across the surface mostly by hopping over single silver atomic sites (Figure $3 \mathrm{c}, \mathrm{f}$ ), and the molecule remains rigid most of the time although occasional flipping of the alternate $\pi$ rings could occur (Supporting Information). These manipulation curves resemble stick-slip style movement ${ }^{33}$ in pulling (attractive tip-molecule interactions). Interestingly, the lateral manipulation signal (Figure 3c,f) from the sideways direction reveals a larger tip height variation than that from the parallel direction. Consequently, averaged manipulation curves provide a smaller force angle $(\phi)$ in moving $6 \mathrm{P}$ along the sideways direction than it does for the parallel direction (Figure $3 \mathrm{~g}$ and Supporting Information). ${ }^{34}$ Note that the aperiodicity in the manipulation curves (Figure $3 \mathrm{c}, \mathrm{f}$ ) is caused by a slight deviation $\left(\sim 1\right.$ to $\left.3^{\circ}\right)$ of the manipulation paths from the exact [110] surface direction ${ }^{11}$ inducing flipping of the $\pi$-rings (Supporting Information). Only single surface-atom site hopping signals are counted for the force measurement and aperiodic manipulation signals are discarded in force analysis. From the STM lateral manipulation signal, the magnitude of $F_{\mathrm{L}}$ required to move the $6 \mathrm{P}$ molecule can be estimated by using a directional cosine relationship $^{34}$

$$
F_{\mathrm{L}}=F_{\mathrm{T}} \cos \phi
$$

where $F_{\mathrm{T}}$ is the total tip-molecule force. When $\phi=90^{\circ}, F_{\mathrm{T}}$ is along the direction normal to the surface and thus equals to the vertical force. When $\phi$ deviates from $90^{\circ}$, the direction of $F_{\mathrm{T}}$ changes but its magnitude can be assumed to remain the same. For the same tip height, the same magnitude of $F_{\mathrm{T}}$ is exerted on the molecule and therefore a smaller force angle will produce a larger lateral force.

In order to clarify the observed phenomenon, we perform STM lateral manipulation at different tip heights between $\sim 2$ and $3 \AA$ for both directions. The measured force angles, $\phi$, are plotted as a function of tip heights in Figure $3 \mathrm{~h}$. Within the measured tip height range, the force angle decreases with increasing tip height for both directions. However, the force angles from the sideways direction appear consistently smaller than those from the parallel direction. Since the vertical tip setpoint (tip height) for the manipulation is the same for both 

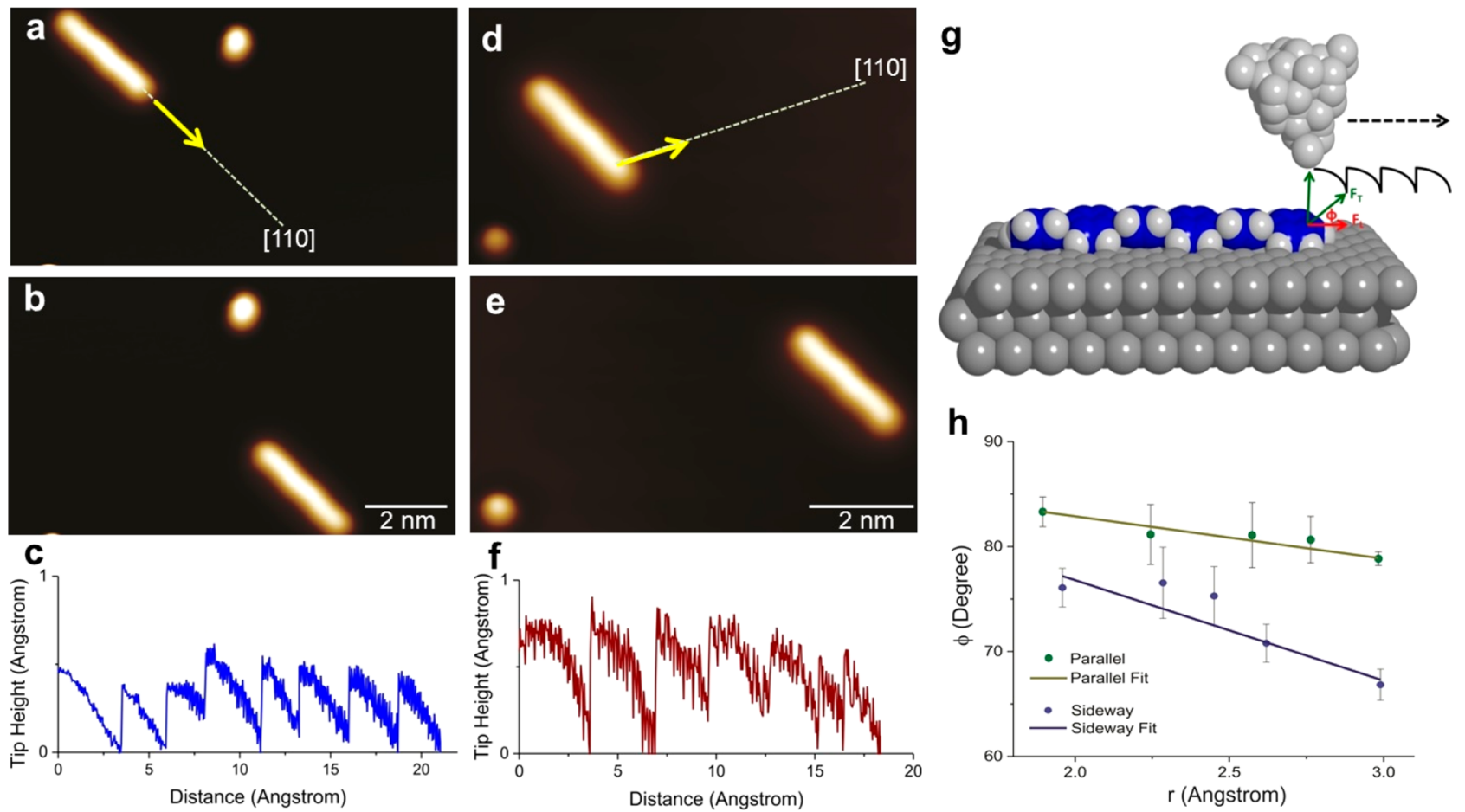

Figure 3. STM image of 6P molecule before (a) and after lateral manipulation (b) along a direction parallel to its long molecular axis (indicated with an arrow) [Image parameters for $(\mathrm{a}, \mathrm{b}): 14.5 \times 6.3 \mathrm{~nm}^{2}, I_{\mathrm{t}}=1.3 \times 10^{-9} \mathrm{~A}, V_{\mathrm{t}}=0.42 \mathrm{~V}$ ]. (c) A typical pulling manipulation curve recorded during a manipulation along parallel direction. (d,e) STM images correspond to before and after lateral manipulation of $6 \mathrm{P}$ along a sideways direction (indicated with an arrow) [Image parameters for $(\mathrm{d}, \mathrm{e}): 11.4 \times 4.9 \mathrm{~nm}^{2}, I_{\mathrm{t}}=1.3 \times 10^{-9} \mathrm{~A}, V_{\mathrm{t}}=0.42 \mathrm{~V}$ ]. (f) A typical pulling manipulation curve recorded during manipulation along sideways direction. [Manipulation parameters for $(\mathrm{c}, \mathrm{f}): R_{\mathrm{t}}=1.2 \mathrm{M} \Omega, V_{\mathrm{t}}=0.12 \mathrm{~V}$ ]. (g) During manipulation with the $S T M$ tip, the sexiphenyl molecule moves in a rest-hop manner producing a typical pulling manipulation signal (black curve). Total and lateral forces $\left(F_{\mathrm{T}}\right.$, and $F_{\mathrm{L}}$ ) as well as the force angle " $\phi$ " are illustrated. (h) Force angle as a function of tip height plots for parallel and sideways directions. The error bars describe the mean statistical distributions and the straight lines are the linear fits.

directions, the magnitude of $F_{\mathrm{T}}$ is the same. Thus, eq 1 can be restructured as

$$
F_{\mathrm{T}}=\frac{F_{\mathrm{Lp}}}{\cos \phi_{\mathrm{p}}}=\frac{F_{\mathrm{Ls}}}{\cos \phi_{\mathrm{s}}}
$$

Here, $F_{L p}$ and $F_{L s}$ are the lateral forces for the parallel and sideways directions while $\phi_{\mathrm{p}}$ and $\phi_{\mathrm{s}}$ are the force angles for these two directions, respectively. From eq 2 , the ratio of the two lateral forces $\left(F_{\mathrm{Ls}} / F_{\mathrm{Lp}}\right)$ will be equal to the ratio of the directional cosine of the two force angles. From the average manipulation signals recorded at different tip heights for the parallel and sideways directions, the $F_{\mathrm{Ls}} / F_{\mathrm{Lp}}$ is determined to be $\sim 1.9 \pm 0.3$. This means that the lateral force required to move the molecule along the sideways direction is about twice as large as compared to the parallel direction in agreement with the direct lateral force measurement performed by $q+$ AFM.

To unravel the observed lateral force phenomenon, we have performed density functional theory (DFT) and classical molecular dynamics (CMD) calculations. Geometrically relaxed DFT calculations including vdW interactions of the 6P adsorbed on a $\operatorname{Ag}(111)$ surface (Supporting Information) confirm the adsorption of the molecule along the $[110]$ surface directions, in agreement with the experimental finding (Figure 4a). The calculations also reveal twisting of alternate $\pi$-rings (indicated with arrows in Figure $4 \mathrm{a}$ ) with a torsional angle of $\sim 18^{\circ}$ (Supporting Information).

Next, using DFT computed adsorption structure of $6 \mathrm{P}$ on $\mathrm{Ag}(111)$ as input, we perform CMD calculations to investigate the lateral forces required to move $6 \mathrm{P}$ along the parallel and sideways directions (Supporting Information). The calculations provide the threshold lateral force to displace the molecule along the parallel direction, $F_{\mathrm{LP}}$, as $\sim 125 \mathrm{pN}$ while that along the sideways direction, $F_{\mathrm{LS}}$, as $\sim 200 \mathrm{pN}$ (Figure $4 \mathrm{~b}$ ). Thus, threshold lateral force along the sideways direction is larger than that of the parallel direction, and the ratio of the lateral forces, $F_{L S} / F_{L P}$, is 1.6. This ratio relatively agrees with the experiments. Additionally, to estimate the effect of temperature fluctuations on the observed threshold lateral force, we have performed five independent simulations over a temperature range of $5-15 \mathrm{~K}$ for both the directions. Variations of \pm 8 and $\pm 10 \mathrm{pN}$ in the magnitude of the lateral force in the sideways and parallel directions are found, respectively, and the ratio of lateral forces remains the same.

The directional lateral force phenomenon observed here can be explained as follow: When the 6P adsorbs on the surface, the molecule-surface attractive interactions lower the potential energy underneath the molecule. When moving toward the parallel direction, only the front $\pi$-ring will encounter the potential barrier imposed by the bare $\operatorname{Ag}(111)$ surface area (depicted with blue color in Figure 4c). However, when the 6P is moved along the sideways directions, all the $\pi$-rings will have to overcome the barrier imposed by the bare $\mathrm{Ag}(111)$ surface (red color in Figure 4c). Therefore, although the number of surface atoms interacting with the molecule is the same in both cases, a higher barrier exists in the sideways direction.

The $\mathrm{q}+\mathrm{AFM}$ and STM experiments as well as CMD simulations described so far is for the threshold lateral force to move the molecule from rest, which may be considered as a 


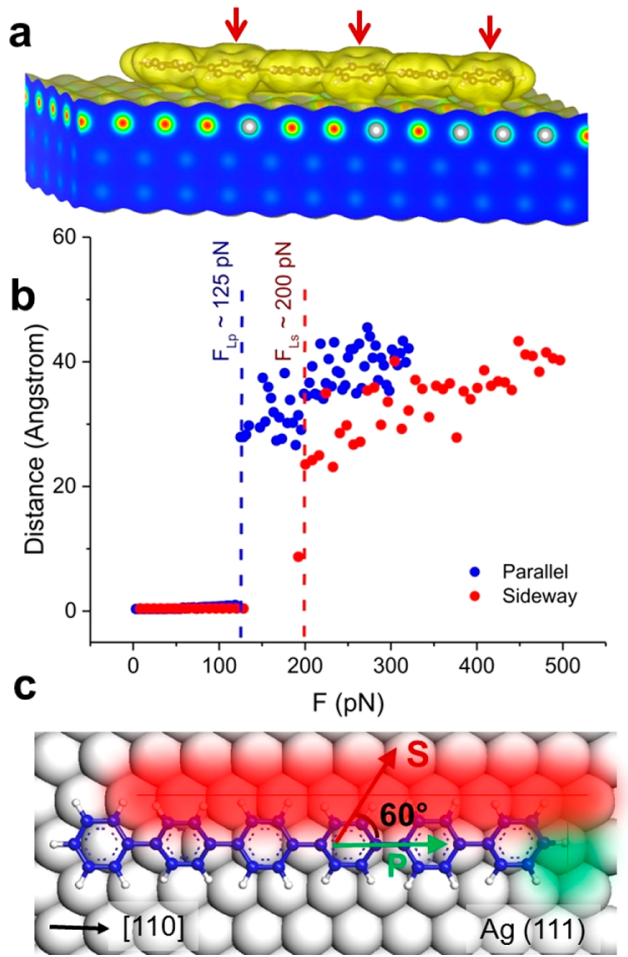

Figure 4. Origin of molecule friction. (a) DFT result of a total density plot of $6 \mathrm{P}$ on $\mathrm{Ag}(111)$ surface. (b) CMD results for distance (traveled by the $6 \mathrm{P}$ molecule over $1 \mathrm{~ns}$ ) as a function of force plots, which reveals the minimum lateral force required to move along the parallel and sideways directions. (c) The adsorption geometry of the $6 \mathrm{P}$ on $\mathrm{Ag}(111)$. Molecular dynamic simulations are performed along the parallel (P) and sideways $(S)$ directions. The green and red colored regions depict the surface areas for the molecule to move one atom site along parallel and sideways, respectively, during lateral displacement.

static friction force for the molecule. In addition, the CMD simulations further reveal the dynamic of $6 \mathrm{P}$ propagation. On $\mathrm{Ag}(111)$ surface $6 \mathrm{P}$ propagates by transferring its energy back and forth between the potential energy ( $\pi$-ring flipping) and the kinetic energy (the translational motion); see SI Movie 1. This is in agreement with a previous report of $6 \mathrm{P}$ movement across the same surface. ${ }^{22}$ Furthermore, the CMD simulations on the sideways direction shows that the molecule first rotates to align its long molecular axis with the underlying close-packed surface direction before moving across the surface along its long parallel axis. This indicates that a higher threshold value of sideways force is required to surmount the additional energetic barriers. In the experiments, the molecule is forced to move with the STM or AFM tip along the sideways direction, and thus manipulation mostly results in displacement of the molecule without rotation, and consequently it costs a higher lateral force than theory. Indeed, rotation of the molecule can be occasionally observed in STM manipulation of $6 \mathrm{P}$ along the sideways direction if the tip is positioned at the end of the molecule (Supporting Information).

In summary, we have determined the lateral forces required to move a $1 \mathrm{D}$ molecule across a $2 \mathrm{D}$ symmetric surface. We have found that the lateral force required to move the $6 \mathrm{P}$ molecule along the sideways direction is almost twice as large as it is to move it along the parallel direction. While AFM is generally used for the force measurements, we have also demonstrated that STM manipulation can be used for the qualitative comparison of lateral forces as well. Our work demonstrates that the lateral force required to move the nanoscale features such as molecules can be influenced by their shapes, and this directional force anisotropy will impact on the fundamental understanding of friction for the nanoscale objects such as in the movement of molecules on a surface at the atomic scale.

\section{ASSOCIATED CONTENT}

\section{SI Supporting Information}

The Supporting Information is available free of charge at https://pubs.acs.org/doi/10.1021/acs.nanolett.0c04974.

Movie showing the movement of molecule in sideways manipulation (MP4)

Movie showing the movement of molecule in parallel manipulation (MP4)

Force measurement with AFM; inflection point test; $\pi$ ring position dependent manipulation; the effect of alternate $\pi$-ring twisting; density functional theory calculations; molecular dynamics simulations; rotation of molecule during manipulation (PDF)

\section{AUTHOR INFORMATION}

\section{Corresponding Author}

Saw Wai Hla - Center for Nanoscale Materials, Nanoscience and Technology Division, Argonne National Laboratory, Lemont, Illinois 60439, United States; Nanoscale and Quantum Phenomena Institute, Department of Physics and Astronomy, Ohio University, Athens, Ohio 45701, United States; orcid.org/0000-0002-6463-9714; Email: hla@ ohio.edu

\section{Authors}

Yuan Zhang - Center for Nanoscale Materials, Nanoscience and Technology Division, Argonne National Laboratory, Lemont, Illinois 60439, United States; Nanoscale and Quantum Phenomena Institute, Department of Physics and Astronomy, Ohio University, Athens, Ohio 45701, United States; Department of Physics, Old Dominion University, Norfolk, Virginia 23529, United States

Daniel J. Trainer - Center for Nanoscale Materials, Nanoscience and Technology Division, Argonne National Laboratory, Lemont, Illinois 60439, United States

Badri Narayanan - Center for Nanoscale Materials, Nanoscience and Technology Division, Argonne National Laboratory, Lemont, Illinois 60439, United States; Department of Mechanical Engineering, University of Louisville, Louisville, Kentucky 40292, United States

Yang Li - Center for Nanoscale Materials, Nanoscience and Technology Division, Argonne National Laboratory, Lemont, Illinois 60439, United States; Nanoscale and Quantum Phenomena Institute, Department of Physics and Astronomy, Ohio University, Athens, Ohio 45701, United States

Anh T. Ngo - Materials Science Division, Argonne National Laboratory, Lemont, Illinois 60439, United States; Department of Chemical Engineering, University of Illinois, Chicago, Illinois 60607, United States

Sushila Khadka - Nanoscale and Quantum Phenomena Institute, Department of Physics and Astronomy, Ohio University, Athens, Ohio 45701, United States

Arnab Neogi - Center for Nanoscale Materials, Nanoscience and Technology Division, Argonne National Laboratory, Lemont, Illinois 60439, United States; Department of 
Mechanical and Industrial Engineering, University of Illinois, Chicago, Illinois 60607, United States

Brandon Fisher - Center for Nanoscale Materials, Nanoscience and Technology Division, Argonne National Laboratory, Lemont, Illinois 60439, United States

Larry A. Curtiss - Materials Science Division, Argonne National Laboratory, Lemont, Illinois 60439, United States; (1) orcid.org/0000-0001-8855-8006

Subramanian K. R. S. Sankaranarayanan - Center for Nanoscale Materials, Nanoscience and Technology Division, Argonne National Laboratory, Lemont, Illinois 60439, United States; Department of Mechanical and Industrial Engineering, University of Illinois, Chicago, Illinois 60607, United States; 다이.org/0000-0002-9708-396X

Complete contact information is available at:

https://pubs.acs.org/10.1021/acs.nanolett.0c04974

\section{Author Contributions \\ OY.Z. and D.J.T. contributed equally.}

\section{Author Contributions}

S.W.H. conceived and designed the experiments. Y.Z., D.J.T., and Y.L. performed the STM, and q+AFM experiments. Y.Z., S.K., Y.L., and S.W.H. analyzed the experimental data. B.F. supported the q+AFM experiments. B.N., S.S., and A.N. performed the molecular dynamics simulations, and A.T.N. and L.A.C. performed the DFT calculations. All the authors discussed the results and commented on the manuscript.

\section{Notes}

The authors declare no competing financial interest.

\section{ACKNOWLEDGMENTS}

The STM experiments and analysis were supported by the U.S. Department of Energy, Office of Science, Office of Basic Energy Sciences, Grant DE-FG02-02ER46012. Use of the Center for Nanoscale Materials, an Office of Science user facility, was supported by the U.S. Department of Energy, Office of Science, Office of Basic Energy Sciences, under Contract No. DE-AC0206CH11357. Y.L. is supported by the U.S. Department of Energy Office of Basic Energy Sciences (SISGR Grant DEFG02-09ER16109). L.A.C. and A.T.N. were supported by the U.S. Department of Energy, Office of Basic Energy Sciences, Materials Science and Engineering. We gratefully acknowledge the computer time from the Argonne National Laboratory Computing Resource Center (LCRC).

\section{REFERENCES}

(1) Ternes, M.; Lutz, C. P.; Hirjibehedin, C. F.; Giessibl, F. J.; Heinrich, A. J. The Force Needed to Move an Atom on a Surface. Science 2008, 319, 1066-1069.

(2) Kawai, S.; Benassi, A.; Gnecco, E.; Söde, H.; Pawlak, R.; Feng, X.; Müllen, K.; Passerone, D.; Pignedoli, C. A.; Ruffieux, P.; Fasel, R.; Meyer, E. Superlubricity of Graphene Nanoribbons on Gold Surfaces. Science 2016, 351, 957-961.

(3) Custance, O.; Perez, R.; Morita, S. Atomic Force Microscopy as a Tool for Atom Manipulation. Nat. Nanotechnol. 2009, 4, 803-810.

(4) Pawlak, R.; Ouyang, W.; Filippov, A. E.; Kalikhman-Razvozov, L.; Kawai, S.; Glatzel, T.; Gnecco, E.; Baratoff, A.; Zheng, Q.; Hod, O.; Urbakh, M.; Meyer, E. Single-Molecule Tribology: Force Microscopy Manipulation of a Porphyrin Derivative on a Copper Surface. ACS Nano 2015, 10, 713-722.

(5) Comtet, J.; Lainé, A.; Niguès, A.; Bocquet, L.; Siria, A. Atomic Rheology of Gold Nanojunctions. Nature 2019, 569, 393-397.
(6) Sheehan, P. E.; Lieber, C. Friction between van der Waals Solids during Lattice Directed Sliding. Nano Lett. 2017, 17, 4116-4121.

(7) Gross, L.; Mohn, F.; Moll, N.; Liljeroth, P.; Meyer, G. The Chemical Structure of a Molecule Resolved by Atomic Force Microscopy. Science 2009, 325, 1110-1114.

(8) Park, J. Y.; Salmeron, M. Fundamental Aspects of Energy Dissipation in Friction. Chem. Rev. 2014, 114, 677-711.

(9) Vazirisereshk, M. R.; Ye, H.; Ye, Z.; Otero-de-la-Roza, A.; Zhao, M.-Q.; Gao, Z.; Charlie Johnson, A. T.; Johnson, E. R.; Carpick, R. W.; Martini, A. Origin of Nanoscale Friction Contrast between Supported Graphene, $\mathrm{MoS}_{2}$, and a Graphene/ $\mathrm{MoS}_{2}$ Heterostructure. Nano Lett. 2019, 19, 5496-5505.

(10) Wolter, B.; Yoshida, Y.; Kubetzka, A.; Hla, S.-W.; von Bergmann, K.; Wiesendanger, R. Spin Friction Observed on the Atomic Scale. Phys. Rev. Lett. 2012, 109, 116102.

(11) Hla, S.-W.; Braun, K.-F.; Rieder, K.-H. Single-Atom Manipulation Mechanisms during a Quantum Corral Construction. Phys. Rev. B: Condens. Matter Mater. Phys. 2003, 67, 201402.

(12) Bhushan, B.; Israelachvili, J. N.; Landman, U. Nanotribology: Friction, Wear and Lubrication at the Atomic Scale. Nature 1995, 374, 607-616.

(13) Hod, O.; Meyer, E.; Zheng, Q.; Urbakh, M. Structural Superlubricity and Ultralow Friction Across the Length Scales. Nature 2018, 563, 485-492.

(14) Mandelli, D.; Tosatti, E. Microscopic Friction Emulators. Nature 2015, 526, 332-333.

(15) Mo, Y.; Turner, K. T.; Szlufarska, I. Friction Laws at the Nanoscale. Nature 2009, 457, 1116-1119.

(16) Luan, B.; Robbins, M. O. The Breakdown of Continuum Models for Mechanical Contacts. Nature 2005, 435, 929-932.

(17) Guerra, R.; Tartaglino, U.; Vanossi, A.; Tosatti, E. Ballistic Nanofriction. Nat. Mater. 2010, 9, 634-637.

(18) Krylov, S. Y.; Frenken, J. W. M. The Physics of Atomic-Scale Friction: Basic Considerations and Open Questions. Phys. Status Solidi B 2014, 251, 711-736.

(19) Ben-David, O.; Rubinstein, S. M.; Fineberg, J. Slip-Stick and the Evolution of Frictional Strength. Nature 2010, 463, 76-79.

(20) Schirmeisen, A.; Weiner, D.; Fuchs, H. Single-Atom Contact Mechanics: From Atomic Scale Energy Barrier to Mechanical Relaxation Hysteresis. Phys. Rev. Lett. 2006, 97, 136101.

(21) Evstigneev, M.; Reimann, P. Force Dependence of Energy Barriers in Atomic Friction and Single-Molecule Force Spectroscopy: Critique of a Critical Scaling Relation. J. Phys.: Condens. Matter 2015, 27,125004 .

(22) Braun, K.-F.; Hla, S.-W. Probing the Conformation of Physisorbed Molecules at the Atomic-Scale using STM Manipulation. Nano Lett. 2005, 5, 73-76.

(23) Zhang, Y.; Wang, S.; Braun, K.-F.; Hla, S.-W. Molecular Flexure and Atom Trapping with Sexiphenyl Molecules by Scanning Tunneling Microscope Manipulation. Surf. Sci. 2018, 678, 215-221.

(24) Koller, G.; Berkebile, S.; Oehzelt, M.; Puschnig, P.; AmbroschDraxl, C.; Netzer, F. P.; Ramsey, M. G. Intra- and Intermolecular Band Dispersion in an Organic Crystal. Science 2007, 317, 351-355.

(25) Matković, A.; Genser, J.; Lüftner, D.; Kratzer, M.; Gajić, R.; Puschnig, P.; Teichert, C. Epitaxy of Highly Ordered Organic Semiconductor Crystallite Networks Supported by Hexagonal Boron Nitride. Sci. Rep. 2016, 6, 38519.

(26) Qian, C.; Sun, J.; Kong, L.-A.; Gou, G.; Zhu, M.; Yuan, Y.; Huang, H.; Gao, Y.; Yang, J. High-Performance Organic Heterojunction Phototransistors Based on Highly Ordered Copper Phthalocyanine/ Para-Sexiphenyl Thin Films. Adv. Funct. Mater. 2017, 27, 1604933.

(27) Hla, S.-W. STM Single Atom/Molecule Manipulation and Its Application to Nanoscience and Technology. J. Vac. Sci. Technol., B: Microelectron. Process. Phenom. 2005, 23, 1351-1360.

(28) Sader, J. E.; Jarvis, S. P. Accurate Formulas for Interaction Force and Energy in Frequency Modulation Force Spectroscopy. Appl. Phys. Lett. 2004, 84, 1801-1803. 
(29) Sader, J. E.; Hughes, B. D.; Huber, F.; Giessibl, F. J. Interatomic Force Laws that Evade Dynamic Measurement. Nat. Nanotechnol. 2018, 13, 1088-1091.

(30) Huber, F.; Giessibl, F. J. Experimental Use of the Inflection Point Test for Force Deconvolution in Frequency Modulation Atomic Force Microscopy to Turn an Ill-Posed Situation into a Well-Posed One by Proper Choice of Amplitude. J. Appl. Phys. 2020, 127, 184301.

(31) Hla, S.-W. Atom-by-Atom Assembly. Rep. Prog. Phys. 2014, 77, 056502.

(32) Hla, S.-W.; Kuhnle, A.; Bartels, L.; Meyer, G.; Rieder, K.-H. Controlled Lateral Manipulation of Single Diiodobenzene Molecules on the $\mathrm{Cu}(111)$ Surface with the Tip of a Scanning Tunnelling Microscope. Surf. Sci. 2000, 454, 1079-1084.

(33) Ben-David, O.; Rubinstein, S. M.; Fineberg, J. Slip-Stick and the Evolution of Frictional Strength. Nature 2010, 463, 76-79.

(34) Deshpande, A.; Braun, K.-F.; Hla, S.-W. Determination of Chemical Specific Atomic Interaction with Scanning Tunneling Microscope. Appl. Phys. Lett. 2011, 99, 221902. 